\title{
Low-frequency critical current noise in graphene Josephson junctions in the open-circuit gate voltage limit
}

\author{
Francesco M. D. Pellegrino ${ }^{1,2}$, Giuseppe Falci ${ }^{1,2,3}$, and Elisabetta Paladino ${ }^{1,2,3, a}$ \\ 1 Dipartimento di Fisica e Astronomia "Ettore Majorana", Università di Catania, Via S. Sofia 64,, Catania 95123, Italy \\ 2 INFN, Sez. Catania, Catania 95123, Italy \\ 3 CNR-IMM, Via S. Sofia 64,, Catania I-95123, Italy
}

Received 27 April 2020 / Accepted 5 January 2021 / Published online 16 April 2021

(C) The Author(s) 2021

\begin{abstract}
We investigate critical current noise in short ballistic graphene Josephson junctions in the opencircuit gate-voltage limit within the McWorther model. We find flicker noise in a wide frequency range and discuss the temperature dependence of the noise amplitude as a function of the doping level. At the charge neutrality point we find a singular temperature dependence $T^{-3}$, strikingly different from the linear dependence expected for short ballistic graphene Josephson junctions under fixed gate voltage.
\end{abstract}

\section{Introduction}

The encapsulation of a graphene sample in hexagonal boron nitride allows the production of devices characterized by ballistic transport features up to room temperature at a micrometer scale for a wide range of carrier concentration [1-3]. Based on this technology, ballistic bipolar graphene Josephson junctions (GJJ) have been realized [4-6]. These high-quality graphene samples with superconducting electrodes in close proximity to the graphene layer with ultra-clean interfaces allow Cooper pairs ballistically roaming over micron scale lengths [7-9]. In a GJJ, a dissipationless supercurrent flows in equilibrium through the normal region via the Andreev reflections at each superconductorgraphene interface. In this system, the Andreev level spectrum, and as a consequence the current-phase relation, depends on the normal phase channel length $(L)$ and on the doping level in graphene layer. In this work, we focus on the short channel limit, $L \ll \xi$, where $\xi$ is the coherence length in the superconductor. This regime has been recently experimentally achieved in [6], and well described within the Dirac-Bogoliubov-de Gennes approach [10]. The tunability of the currentphase relation by varying the doping level has allowed the realization of voltage-controlled transmon, also known as a gatemon [11,12].

In this manuscript, we investigate the critical current noise in short ballistic GJJ within the McWorther model [13] commonly used to describe noise induced by electron traps in oxide substrates [14-18]. Charge traps act as independent generation-recombination centers, which are described as random telegraph processes. Due

a e-mail: elisabetta.paladino@dfa.unict.it (corresponding author) to proximitized superconductivity of the normal metal forming the junction, fluctuations of carrier density in the graphene insert induce fluctuations of Andreev levels, resulting in noise in the critical current of the ballistic GJJ [19].

Here, we address the open-circuit gate-voltage limit, excluding any charge flow between the graphene layer and the metal gate via the external circuit. This regime is complementary to the fixed graphene-to-metal-gate voltage-drop regime where charge flow through the circuit allows readjustment of the number of carriers in graphene after each trapping/recombination process. In Ref. [19] the fixed- $V_{\mathrm{G}}$ regime was considered under the assumption of instantaneous equilibration of carriers in graphene after each tunneling process. In an equivalent circuit description, in the present manuscript we address the infinite external resistance limit of the bias circuit, whereas the zero resistance limit was considered in Ref. [19]. In the following, we will refer to these two regimes as the "open-circuit" and "fixed gate voltage" operating conditions, respectively. We demonstrate that, similarly to the fixed gate voltage regime, noise in the critical current due to fluctuations of the carrier density in graphene depends on frequency, $\omega$, as $S_{I_{c}}(\omega)=\mathcal{A}(T, \mu) / \omega$, where $\mu$ is the chemical potential in graphene. For large doping we find that $\mathcal{A}(T, \mu)$ is independent on the operating regime, or equivalently on the equilibration time in graphene, as expected. At the charge neutrality point (CNP) instead we find qualitative differences leading to striking consequences in the low-temperature behavior of the noise amplitude $\mathcal{A}(T, \mu=0)$. We find that in the open-circuit limit the noise amplitude diverges as $\mathcal{A}(T, 0) \propto T^{-3}$, whereas for fixed gate voltage it is $\mathcal{A}_{V_{\mathrm{G}}}(T, 0) \propto T$. 


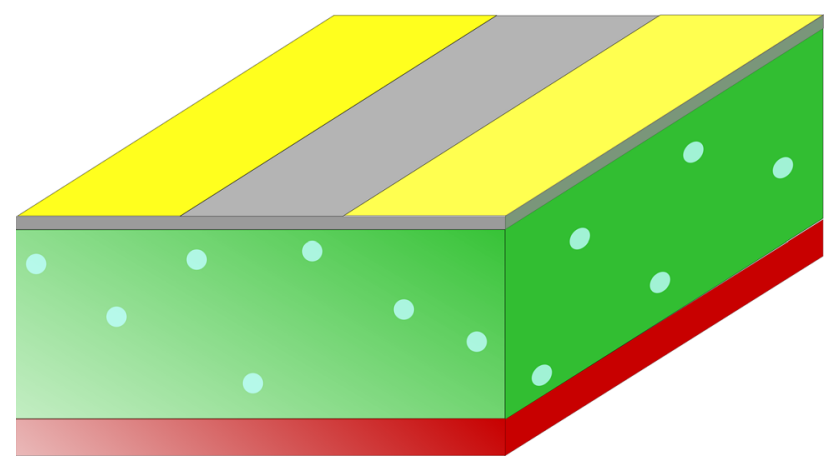

Fig. 1 Schematic of the device (side view). The device consists of a metal gate (red), a substrate (green), a monolayer graphene (gray), and two superconducting electrodes (yellow). Within the substrate, the cyan circles represent the electron traps

\section{Model}

Here we introduce the model which relates the charge carrier density in graphene to charge traps in the substrate. We refer to the simple device shown in Fig. 1, formed by a monolayer graphene on a substrate hosting electron traps placed on top of a metal gate. The metal-substrate interface is at $z=0$, the graphene layer is treated as a two-dimensional system at $z=d$ and it is partially covered by two superconducting electrodes. The substrate width $d$ is much smaller than both longitudinal sizes along $\hat{x}$ and $\hat{y}$ directions.

Carrier density fluctuations are due to charge trapping and release processes between graphene and carrier traps in the underlying substrate, and the trapping and recombinations are considered as discrete Markov processes [20]. The occupancy number $X(i, t)$ of the trap labeled by the index $i$ at time $t$ is a random variable. Each trap can be empty $(X(i, t)=0)$ or occupied by a single electron $(X(i, t)=1)$, and it randomly switches between these states with time-independent rates (stationary process). The conditional probability that the trap $i$ at time $t$ has the occupation number $X(i, t)$ if the trap $j$ at time $t_{0}$ has the occupation number $X\left(j, t_{0}\right)$ is written as

$$
\begin{aligned}
& P\left[X(i, t) \mid X\left(j, t_{0}\right)\right]=\delta_{i, j} p_{i}\left[X\left(i, t-t_{0}\right) \mid X(i, 0)\right] \\
& \quad+w_{i}(X(i, 0))
\end{aligned}
$$

where $w_{i}(X(i, 0))$ is the stationary probability of trap $i$ which depends on the initial occupation $X(i, 0)$ as follows $w_{i}(1)=f_{i}$ and $w_{i}(0)=1-f_{i}$, and the Kronecker delta appears because different traps are uncorrelated. The matrix $p_{i}$ is expressed as [19-21]

$$
\begin{aligned}
p_{i}[X(i, t) \mid X(i, 0)] & =\left[\begin{array}{c}
p_{i}[0 \mid 0](t) \\
p_{i}[1 \mid 0](1) \\
p_{i}[0](t) \\
p_{i}[1 \mid 1](t)
\end{array}\right] \\
& =\left[\begin{array}{cc}
f_{i} & -\left(1-f_{i}\right) \\
-f_{i} & 1-f_{i}
\end{array}\right] e^{-\gamma_{i} t},
\end{aligned}
$$

where $\gamma_{i}=\lambda_{i, 00}+\lambda_{i, 11}$ is the switching rate between the two states of the stochastic process expressed in terms of the transition rates for $0 \rightarrow 1$ and $1 \rightarrow 0$ processes, $\lambda_{i, 00}$ and $\lambda_{i, 11}$, respectively. Due to Markovianity, the multi-time correlators reduce to two-points correlation function

$$
\begin{aligned}
& P\left[X\left(i_{N}, t_{N}\right) \mid X\left(i_{N-1}, t_{N-1}\right) ; \ldots ; X\left(i_{0}, t_{0}\right)\right] \\
& \quad=P\left[X\left(i_{N}, t_{N}\right) \mid X\left(i_{N-1}, t_{N-1}\right)\right] .
\end{aligned}
$$

The density of populated traps per unit volume and energy, $\mathcal{N}_{\mathrm{T}}(\epsilon, \boldsymbol{R}, t)$, fluctuates around its average value $\mathcal{N}_{\text {T0 }}$. Assuming that trap $i$ is located at position $\boldsymbol{R}_{i}$ and that the energy of the occupied trap is $\epsilon_{i}$ (evaluated with respect to the $\mathrm{CNP}$ ), the average value can be expressed as

$$
\mathcal{N}_{\mathrm{T} 0}(\epsilon, \boldsymbol{R})=\sum_{i=1}^{M_{\mathrm{T}}} \delta\left(\epsilon-\epsilon_{i}\right) \delta\left(\boldsymbol{R}-\boldsymbol{R}_{i}\right) f_{i}
$$

and the fluctuations as

$$
\begin{array}{r}
\delta \mathcal{N}_{\mathrm{T}}(\epsilon, \boldsymbol{R}, t)=\mathcal{N}_{\mathrm{T}}(\epsilon, \boldsymbol{R}, t)-\mathcal{N}_{\mathrm{T} 0}(\epsilon, \boldsymbol{R}) \\
=\sum_{i=1}^{M_{\mathrm{T}}} \delta\left(\epsilon-\epsilon_{i}\right) \delta\left(\boldsymbol{R}-\boldsymbol{R}_{i}\right)\left[X(i, t)-f_{i}\right],
\end{array}
$$

where $M_{\mathrm{T}}$ is the total amount of traps. From here on, we assume that the stationary probability coincides with the equilibrium occupation function $f_{i}=$ $f\left(\epsilon_{i}, \boldsymbol{R}_{i}\right)=f_{\mathrm{D}}\left(\epsilon_{i}-\mu_{0}\right)$, where $f_{\mathrm{D}}(x)=1 /\{1+$ $\left.\exp \left[x /\left(k_{\mathrm{B}} T\right)\right]\right\}$ is the Fermi-Dirac distribution function and $\mu_{0}$ is the Fermi level. Under these conditions, the average density of populated traps per unit volume and energy and the multi-time correlators read

$$
\begin{aligned}
& \left\langle\delta \mathcal{N}_{\mathrm{T}}(\epsilon, \boldsymbol{R}, t)\right\rangle=0, \\
& \left\langle\delta \mathcal{N}_{\mathrm{T}}\left(\epsilon_{1}, \boldsymbol{R}_{1}, t_{1}\right) \delta \mathcal{N}_{\mathrm{T}}\left(\epsilon_{0}, \boldsymbol{R}_{0}, t_{0}\right)\right\rangle \\
& =\delta\left(\boldsymbol{R}_{1}-\boldsymbol{R}_{0}\right) \delta\left(\epsilon_{1}-\epsilon_{0}\right) \mathcal{D}\left(\epsilon_{0}, \boldsymbol{R}_{0}\right) f_{\mathrm{D}}\left(\epsilon_{0}-\mu_{0}\right) \\
& {\left[1-f_{\mathrm{D}}\left(\epsilon_{0}-\mu_{0}\right)\right] \exp \left[-\gamma\left(\epsilon_{0}, \boldsymbol{R}_{0}\right)\left(t_{1}-t_{0}\right)\right],} \\
& \left\langle\delta \mathcal{N}_{\mathrm{T}}\left(\epsilon_{2}, \boldsymbol{R}_{2}, t_{2}\right) \delta \mathcal{N}_{\mathrm{T}}\left(\epsilon_{1}, \boldsymbol{R}_{1}, t_{1}\right) \delta \mathcal{N}_{\mathrm{T}}\left(\epsilon_{0}, \boldsymbol{R}_{0}, t_{0}\right)\right\rangle \\
& =\delta\left(\boldsymbol{R}_{2}-\boldsymbol{R}_{1}\right) \delta\left(\boldsymbol{R}_{1}-\boldsymbol{R}_{0}\right) \\
& \left.\quad \times \delta\left(\epsilon_{2}-\epsilon_{1}\right) \delta\left(\epsilon_{1}-\epsilon_{0}\right) \mathcal{D}\left(\epsilon_{0}, \boldsymbol{R}_{0}\right)\right) f_{\mathrm{D}}\left(\epsilon_{0}-\mu_{0}\right) \\
& {\left[1-f_{\mathrm{D}}\left(\epsilon_{0}-\mu_{0}\right)\right]\left[1-2 f_{\mathrm{D}}\left(\epsilon_{0}-\mu_{0}\right)\right]} \\
& \quad \times \exp \left[-\gamma\left(\epsilon_{0}, \boldsymbol{R}_{0}\right)\left(t_{2}-t_{0}\right)\right] \\
& \left\langle\delta \mathcal{N}_{\mathrm{T}}\left(\epsilon_{3}, \boldsymbol{R}_{3}, t_{3}\right)\right. \\
& \left.\delta \mathcal{N}_{\mathrm{T}}\left(\epsilon_{2}, \boldsymbol{R}_{2}, t_{2}\right) \delta \mathcal{N}_{\mathrm{T}}\left(\epsilon_{1}, \boldsymbol{R}_{1}, t_{1}\right) \delta \mathcal{N}_{\mathrm{T}}\left(\epsilon_{0}, \boldsymbol{R}_{0}, t_{0}\right)\right\rangle \\
& =\left\langle\delta \mathcal{N}_{\mathrm{T}}\left(\epsilon_{3}, \boldsymbol{R}_{3}, t_{3}\right) \delta \mathcal{N}_{\mathrm{T}}\left(\epsilon_{2}, \boldsymbol{R}_{2}, t_{2}\right)\right\rangle \\
& \left\langle\delta \mathcal{N}_{\mathrm{T}}\left(\epsilon_{1}, \boldsymbol{R}_{1}, t_{1}\right) \delta \mathcal{N}_{\mathrm{T}}\left(\epsilon_{0}, \boldsymbol{R}_{0}, t_{0}\right)\right\rangle \\
& \quad+\delta\left(\boldsymbol{R}_{3}-\boldsymbol{R}_{2}\right) \delta\left(\boldsymbol{R}_{2}-\boldsymbol{R}_{1}\right) \delta\left(\boldsymbol{R}_{1}-\boldsymbol{R}_{0}\right) \\
& \delta\left(\epsilon_{3}-\epsilon_{2}\right) \delta\left(\epsilon_{2}-\epsilon_{1}\right) \delta\left(\epsilon_{1}-\epsilon_{0}\right) \\
& \quad \times \mathcal{D}\left(\epsilon_{0}, \boldsymbol{R}_{0}\right) f_{\mathrm{D}}\left(\epsilon_{0}-\mu_{0}\right)\left[1-f_{\mathrm{D}}\left(\epsilon_{0}-\mu_{0}\right)\right] \\
& {\left[1-2 f_{\mathrm{D}}\left(\epsilon_{0}-\mu_{0}\right)\right]^{2} \exp \left[-\gamma\left(\epsilon_{0}, \boldsymbol{R}_{0}\right)\left(t_{3}-t_{0}\right)\right] .}
\end{aligned}
$$


Here, $\mathcal{D}(\epsilon, \boldsymbol{R})=\sum_{i=1}^{M_{\mathrm{T}}} \delta\left(\epsilon-\epsilon_{i}\right) \delta\left(\boldsymbol{R}-\boldsymbol{R}_{i}\right)$ is the number of trap states per unit volume and energy at position $\boldsymbol{R}$ for energy $\epsilon$. We assumed that the switching rates $\gamma$ depend only on the trap distance from the graphene layer as $[13,15]$

$$
\gamma(\epsilon, \boldsymbol{R})=\gamma_{0} \exp \left[-|z-d| / \ell_{0}\right]
$$

usually the width of the substrate is $d \sim 100 \mathrm{~nm}$, and typical orders of magnitude of the tunneling parameters are $\gamma_{0} \sim 10^{10} \mathrm{~s}^{-1}$ and $\ell_{0} \sim 1-20 \AA$, respectively $[15,23]$. In addition, we assume that traps are homogeneously distribuited in the substrate, $\mathcal{D}(\epsilon, \boldsymbol{R}) \rightarrow \mathcal{D}(\epsilon)$. Under these conditions, the deviations of the total number of electrons in the traps from its equilibrium value is

$$
\delta N_{\mathrm{T}}(t)=\int d \boldsymbol{r} \int_{0}^{d} d z \int_{-\Lambda}^{\Lambda} d \epsilon \delta \mathcal{N}_{\mathrm{T}}(\epsilon, \boldsymbol{R}, t)
$$

where $\boldsymbol{R}=(\boldsymbol{r}, z)$, and $\Lambda$ is the cut-off energy. In the open-circuit limit charge flow between the graphene layer and the metal gate via the external circuit is not possible. Thus generation-recombination processes in the traps result in fluctuations of the total number of carriers on the graphene layer opposite to fluctuations of the number of electrons in the traps, i.e. $\delta N(t)=-\delta N_{\mathrm{T}}(t)$. We investigate the critical current noise in short ballistic GJJs with the Dirac-Bogoliubovde Gennes approach of Ref. [10]. The resulting critical current, $I_{\mathrm{c}}$, depends on the doping level $\mu$ which is, in turn, related to the carrier density. Thus $\delta \mu \approx$ $\left(d \mu_{0} / d n_{0}\right) \delta N$, where $\mu_{0}$ and $n_{0}$ are respectively the chemical potential and the carrier density in graphene at equilibrium. Treating fluctuations of the doping level as an adiabatic perturbation of the Andreev levels, critical current fluctuations can be expressed as $\delta I_{\mathrm{c}}(t)=$ $I_{\mathrm{c}}(t)-\left\langle I_{\mathrm{c}}(t)\right\rangle$, where

$$
\begin{gathered}
I_{\mathrm{c}}(t) \approx I_{\mathrm{c}}\left(\mu_{0}\right)-\frac{d I_{\mathrm{c}}}{d \mu_{0}} \varepsilon_{\mathrm{T}} \delta N_{\mathrm{T}}(t) \\
+\frac{1}{2} \frac{d^{2} I_{\mathrm{c}}}{d \mu_{0}^{2}} \varepsilon_{\mathrm{T}}^{2}\left[\delta N_{\mathrm{T}}(t)\right]^{2}
\end{gathered}
$$

with $\varepsilon_{\mathrm{T}}=\frac{e^{2}}{S C_{\mathrm{Q}}}, C_{\mathrm{Q}}=e^{2} \frac{d n_{0}}{d \mu_{0}}$ is the quantum capacitance [22] and $S$ is the area of the graphene stripe in normal phase. The power spectrum of the critical current takes the following analytical form

$$
\begin{aligned}
\mathcal{S}_{I_{\mathrm{c}}}(\omega)= & \int_{0}^{\infty} \frac{d t}{\pi} \cos (\omega t)\left\langle\delta I_{\mathrm{c}}(t) \delta I_{\mathrm{c}}(0)\right\rangle \\
= & \frac{\mathcal{A}(\mu, T)}{\omega}= \\
= & {\left[\left(\frac{d I_{\mathrm{c}}}{d \mu_{0}}\right)^{2} F_{0}-\left(\frac{d I_{\mathrm{c}}}{d \mu_{0}}\right)\left(\frac{d^{2} I_{\mathrm{c}}}{d \mu_{0}^{2}}\right) \varepsilon_{\mathrm{T}} F_{1}+\left(\frac{d^{2} I_{\mathrm{c}}}{d \mu_{0}^{2}}\right)^{2} \frac{\varepsilon_{\mathrm{T}}^{2}}{4} F_{2}\right] } \\
& \varepsilon_{\mathrm{T}}^{2} \frac{S \ell_{0}}{2 \omega} \mathcal{W}(\omega),
\end{aligned}
$$

where

$$
\begin{gathered}
F_{j}=\int_{-\Lambda}^{\Lambda} d \epsilon \mathcal{D}(\epsilon) f_{\mathrm{D}}\left(\epsilon-\mu_{0}\right)\left[1-f_{\mathrm{D}}\left(\epsilon-\mu_{0}\right)\right] \\
{\left[1-2 f_{\mathrm{D}}\left(\epsilon-\mu_{0}\right)\right]^{j}} \\
\mathcal{W}(\omega)=\frac{2}{\pi}\left[\arctan \left(e^{d / \ell_{0}} \omega / \lambda_{0}\right)-\arctan \left(\omega / \lambda_{0}\right)\right] .
\end{gathered}
$$

In the frequency range $\gamma_{0} e^{-d / \ell_{0}} \ll \omega \ll \gamma_{0}$, it is easy to verify that $\mathcal{W}(\omega) \approx 1$, thus the critical current power spectrum exhibits flicker noise. The power spectrum in Eq. (13) is the main achievement of this work. This expression has the same form obtained in the fixed gatevoltage limit in Ref. [19]. The only difference is that in the open-circuit regime $\varepsilon_{\mathrm{T}}=e^{2} /\left(S C_{\mathrm{Q}}\right)$ enters the noise amplitude instead of $\varepsilon_{\mathrm{Q}}=e^{2} /\left[S\left(C_{\mathrm{Q}}+C_{\mathrm{g}}\right)\right]$, where $C_{\mathrm{g}}=$ $\epsilon_{\mathrm{r}} /(4 \pi d)$ is the geometric capacitance $\left(\epsilon_{\mathrm{r}}\right.$ is the relative dielectric constant of the substrate). For large doping, the quantum capacitance is approximately given by $C_{\mathrm{Q}} \approx \frac{2 e^{2}}{\pi \hbar^{2} v_{\mathrm{D}}^{2}}\left|\mu_{0}\right| \gg C_{\mathrm{g}}$ (where $v_{\mathrm{D}} \sim 10^{6} \mathrm{~m} / \mathrm{s}$ ), therefore $\varepsilon_{\mathrm{Q}} \approx \varepsilon_{\mathrm{T}}$ and the noise amplitude $\mathcal{A}(\mu, T)$ does not depend on the operating condition. In other words, within the McWorther model, the critical current power spectrum for large doping does not depend on the thermalization time of the carrier density in graphene. For zero doping instead, the quantum capacitance is written as $C_{\mathrm{Q}} \approx \frac{2 \ln (2) e^{2}}{\pi \hbar^{2} v_{\mathrm{D}}^{2}} k_{\mathrm{B}} T \ll C_{\mathrm{g}}$, which leads to $\varepsilon_{\mathrm{Q}} \ll \varepsilon_{\mathrm{T}}$. This implies a substantial difference in the critical current spectrum in the two operating conditions. In particular, the temperature dependence is qualitatively different. For zero doping it is $d I_{\mathrm{c}} /\left.d \mu_{0}\right|_{\mu_{0}=0}=0$ [10], thus Eq. (13) reduces to

$$
\begin{aligned}
\mathcal{S}_{I_{\mathrm{c}}}(\omega) & \approx\left(\frac{d^{2} I_{\mathrm{c}}}{d \mu_{0}^{2}}\right)^{2} \frac{\varepsilon_{\mathrm{T}}^{4} F_{2} \ell_{0} S}{8 \omega} \\
& =\frac{\mathcal{A}(0, T)}{\omega} .
\end{aligned}
$$

The temperature dependence is included in $\varepsilon_{\mathrm{T}}$ and $F_{2}$. For low-temperatures Eq. (16) gives $\mathcal{A}(0, T) \propto T^{-3}$. This singular behavior arises from $\varepsilon_{\mathrm{T}} \propto 1 / C_{\mathrm{Q}} \propto 1 / T$ and from $F_{2} \approx \mathcal{D}(0) k_{\mathrm{B}} T / 3$, valid for a smooth energy dependence of the density of trap states $\mathcal{D}(\epsilon)$. The singular temperature behaviour of the noise amplitude is a striking consequence of the open-circuit limit. In the fixed gate voltage regime instead the energy scale $\varepsilon_{\mathrm{Q}}$ tends to the constant value $\varepsilon_{\mathrm{Q}} \approx e^{2} /\left(S C_{\mathrm{g}}\right)$ for low temperatures and the noise amplitude depends linearly on $T$ [19].

\section{Conclusions}

In this manuscript, we have studied critical current noise in short ballistic GJJ based on the phenomeno- 
logical McWhorter model [13] in the open-circuit limit and compared with the fixed gate voltage regime investigated in Ref. [19]. We have found that in both operating conditions there is a wide frequency range [15,23], $2 \pi \times 10^{-10} \mathrm{~s}^{-1} \ll f \ll 2 \pi \times 10^{10} \mathrm{~s}^{-1}$, where the critical current spectrum displays the characteristic flicker noise behavior, $\propto 1 / f$. In the large doping regime, the temperature dependence of the power spectrum turns out not to depend on the operating regime, or equivalently on the charge equilibration time in graphene, as expected. At the CNP instead, qualitative differences emerge leading to striking consequences in the low-temperature behavior of the noise amplitude. In the open circuit limit $\mathcal{A}(T, \mu=0)$ exhibits a singular temperature dependence, unlike the fixed gate-voltage regime which implies a linear temperature dependence. Our results suggest a viable experimental validation of the phenomenological McWorther noise mechanism in short-ballistic GJJ by performing critical current noise measurements in open or closed gate voltage circuits. The ultra-sensitivity of the power spectrum close to the CNP suggests the need for a microscopic modelization of the critical current noise mechanism. The resulting spectrum may have relevant implications on the coherent time evolution of graphene-based gatemons, similarly to qubit $[16,24]$ and qutrit $[25]$ realized with conventional Josephson junctions.

Acknowledgements This work has been supported by the Universitá degli Studi di Catania, "Linea di intervento 2" of Dipartimento di Fisica e Astronomia "Ettore Majorana", Piano di Incentivi per la Ricerca di Ateneo 2016/2018, linea di intervento "Chance" and 2020-2022, Project Q-ICT.

Funding Information Open access funding provided by Università degli Studi di Catania within the CRUI-CARE Agreement.

\section{Author contribution statement}

All the authors conceived the work, agreed on the approach to pursue, analysed and discussed the results. F.M.D.P. performed the calculations, EP and GF supervised the work.

Open Access This article is licensed under a Creative Commons Attribution 4.0 International License, which permits use, sharing, adaptation, distribution and reproduction in any medium or format, as long as you give appropriate credit to the original author(s) and the source, provide a link to the Creative Commons licence, and indicate if changes were made. The images or other third party material in this article are included in the article's Creative Commons licence, unless indicated otherwise in a credit line to the material. If material is not included in the article's Creative Commons licence and your intended use is not permitted by statutory regulation or exceeds the permitted use, you will need to obtain permission directly from the copyright holder. To view a copy of this licence, visit http://creativecomm ons.org/licenses/by/4.0/.

\section{References}

1. C.R. Dean, A.F. Young, I. Meric, C. Lee, L. Wang, S. Sorgenfrei, K. Watanabe, T. Taniguchi, P. Kim, K.L. Shepard, J. Hone, Nat. Nanotech. 5, 722-726 (2010)

2. A.S. Mayorov, R.V. Gorbachev, S.V. Morozov, L. Britnell, R. Jalil, L.A. Ponomarenko, P. Blake, K.S. Novoselov, K. Watanabe, T. Taniguchi, A.K. Geim, Nano Lett. 11, 2396-2399 (2011)

3. L. Wang, I. Meric, P.Y. Huang, Q. Gao, Y. Gao, H. Tran, T. Taniguchi, K. Watanabe, L.M. Campos, D.A. Muller, J. Guo, P. Kim, J. Hone, K.L. Shepard, C.R. Dean, Science 342, 614 (2013)

4. I.V. Borzenets, F. Amet, C.T. Ke, A.W. Draelos, M.T. Wei, A. Seredinski, K. Watanabe, T. Taniguchi, Y. Bomze, M. Yamamoto, S. Tarucha, G. Finkelstein, Phys. Rev. Lett. 117, 237002 (2017)

5. G. Nanda, J.L. Aguilera-Servin, P. Rakyta, A. Kormányos, R. Kleine, D. Koelle, K. Watanabe, T. Taniguchi, L.M.K. Vandersypen, S. Goswami, Nano. Lett. 17, 3396-3401 (2017)

6. J. Park, J.H. Lee, G.-H. Lee, Y. Takane, K.-I. Imura, T. Taniguchi, K. Watanabe, H.-J. Lee, Phys. Rev. Lett. 120, 077701 (2018)

7. M. Ben Shalom, M.J. Zhu, V.I. Fal'ko, A. Mishchenko, A.V. Kretinin, K.S. Novoselov, C.R. Woods, K. Watanabe, T. Taniguchi, A.K. Geim, J.R. Prance, Nat. Phys. 12, 318-322 (2016)

8. V.E. Calado, S. Goswami, G. Nanda, M. Diez, A.R. Akhmerov, K. Watanabe, T. Taniguchi, T.M. Klapwijk, L.M.K. Vandersypen, Nat. Nano. 10, 761 (2015)

9. C.D. English, D.R. Hamilton, C. Chialvo, I.C. Moraru, N. Mason, D.J. Van Harlingen, Phys. Rev. B. 94, 115435 (2016)

10. M. Titov, C.W.J. Beenakker, Phys. Rev. B. 74(R), $041401(2006)$

11. J.I.-J. Wang, D. Rodan-Legrain, L. Bretheau, D.L. Campbell, B. Kannan, D. Kim, M. Kjaergaard, P. Krantz, G.O. Samach, F. Yan, J.L. Yoder, K. Watanabe, T. Taniguchi, T.P. Orlando, S. Gustavsson, P. Jarillo-Herrero, W.D. Oliver, Nat. Nanotech. 14, 120 125 (2018)

12. F.M.D. Pellegrino, G. Falci, E. Paladino, Proceedings 12, 33 (2019)

13. A. L. McWhorter, Semiconductor surface physics, R. H. Kingston (University of Philadelphia Press, Philadelphia, PA, 1957), p. 207

14. F.N. Hooge, T.G.M. Kleinpenning, L.K.J. Vandamme, Rep. Prog. Phys. 44, 479-532 (1981)

15. A.A. Balandin, Nat. Nano. 8, 549 (2013)

16. E. Paladino, M.Y. Galperin, G. Falci, B.L. Altshuler, Rev. Mod. Phys. 86, 361 (2014)

17. A. D'Arrigo, A. Mastellone, E. Paladino, G. Falci, New J. Phys. 10, 115006 (2008)

18. E. Paladino, A. Mastellone, A. D'Arrigo, G. Falci, Phys. Rev. B. 81, 052502 (2010)

19. F.M.D. Pellegrino, G. Falci, E. Paladino, Commun. Phys. 3, 6 (2020) 
20. S. Kogan, Electronic Noise and Fluctuations in Solids (Cambridge University Press, Cambridge, UK, 1996), p. 118

21. F.M.D. Pellegrino, G. Falci, E. Paladino, J. Stat. Mech. 2019, 094015 (2019)

22. A.H. Castro Neto, F. Guinea, N.M.R. Peres, K.S. Novoselov, A.K. Geim, Rev. Mod. Phys. 81, 109 (2009)

23. S.T. Hsu, Solid-State Electron. 13, 843-855 (1970)
24. E. Paladino, L. Faoro, G. Falci, Adv. Sol. State Phys. 43, 747 (2003)

25. G. Falci, P.G. Di Stefano, A. Ridolfo, A. D'Arrigo, G.S. Paraoanu, and E. Paladino Fortschr. Phys. 65, 1600077 (2017) 